\title{
A Complete Description of Comparison Meaningful Functions
}

\author{
Jean-Luc MARICHAL Radko MESIAR \\ Tatiana RÜCKSCHLOSSOVÁ
}

Submitted 11 March 2002, Revised 27 November 2003

\begin{abstract}
Comparison meaningful functions acting on some real interval $E$ are completely described as transformed coordinate projections on minimal invariant subsets. The case of monotone comparison meaningful functions is further specified. Several already known results for comparison meaningful functions and invariant functions are obtained as consequences of our description.
\end{abstract}

Key words: comparison meaningful function, invariant function, ordinal scale. 2000 Mathematics Subject Classification: Primary 39B22, Secondary 91E45.

\section{Introduction}

Measurement theory (see e.g. $[6,14]$ ) studies, among others, the assignments to each measured object of a real number so that the ordinal structure of discussed objects is preserved. When aggregating several observed objects, their aggregation is often also characterized by a real number, which can be understood as a function of numerical characterizations of fused objects. A sound approach to such aggregation cannot lead to contradictory results depending on the actual scale (numerical evaluation of objects) we are dealing with. This fact was a key motivation for Orlov [11] when introducing comparison meaningful functions. Their strengthening to invariant functions was proposed by Marichal and Roubens [9]. The general structure of invariant functions (and of monotone invariant functions) is now completely known from recent works of Ovchinnikov [12], Ovchinnikov and Dukhovny [13], Marichal [7], Bartłomiejczyk and Drewniak [2], and Mesiar and Rückschlossová [10]. Moreover, comparison meaningful functions were already characterized in some special cases, e.g., when they are continuous; see Yanovskaya [16] and Marichal [7]. However, a complete description of all comparison meaningful functions was still missing. This gap is now filled by the present paper, which is organized as follows. In the next section, we give some preliminaries and recall some known results. In Section 3, a complete description of comparison meaningful functions is given, while in Section 4 we describe all monotone comparison meaningful functions. 


\section{Preliminaries}

Let $E \subseteq \mathbb{R}$ be a nontrivial convex set and set $e_{0}:=\inf E, e_{1}:=\sup E$, and $E^{\circ}:=$ $E \backslash\left\{e_{0}, e_{1}\right\}$. Let $n \in \mathbb{N}$ be fixed and set $[n]:=\{1, \ldots, n\}$. Denote also by $\Phi(E)$ the class of all automorphisms (nondecreasing bijections) $\phi: E \rightarrow E$, and for $x=\left(x_{1}, \ldots, x_{n}\right) \in E^{n}$ put $\phi(x):=\left(\phi\left(x_{1}\right), \ldots, \phi\left(x_{n}\right)\right)$.

Following the earlier literature, we introduce the next notions and recall a few results.

Definition 2.1 ([9]). A function $f: E^{n} \rightarrow E$ is invariant if, for any $\phi \in \Phi(E)$ and any $x \in E^{n}$, we have $f(\phi(x))=\phi(f(x))$.

Definition $2.2([1,11,16])$. A function $f: E^{n} \rightarrow \mathbb{R}$ is comparison meaningful if, for any $\phi \in \Phi(E)$ and any $x, y \in E^{n}$, we have

$$
f(x)\left\{\begin{array}{l}
< \\
=
\end{array}\right\} f(y) \Rightarrow f(\phi(x))\left\{\begin{array}{c}
< \\
=
\end{array}\right\} f(\phi(y)) .
$$

Definition $2.3([1,5])$. A function $f: E^{n} \rightarrow \mathbb{R}$ is strongly comparison meaningful if, for any $\phi_{1}, \ldots, \phi_{n} \in \Phi(E)$ and any $x, y \in E^{n}$, we have

$$
f(x)\left\{\begin{array}{l}
< \\
=
\end{array}\right\} f(y) \Rightarrow f(\phi(x))\left\{\begin{array}{c}
< \\
=
\end{array}\right\} f(\phi(y))
$$

where here the notation $\phi(x)$ means $\left(\phi_{1}\left(x_{1}\right), \ldots, \phi_{n}\left(x_{n}\right)\right)$.

Definition 2.4 ([2]). A nonempty subset $B$ of $E^{n}$ is called invariant if $\phi(B) \subseteq B$ for any $\phi \in \Phi(E)$, where $\phi(B)=\{\phi(x) \mid x \in B\}$. Moreover, an invariant subset $B$ of $E^{n}$ is called minimal invariant if it does not contain any proper invariant subset.

It can be easily proved that $B \subseteq E^{n}$ is invariant if and only if its characteristic function $\mathbf{1}_{B}: E^{n} \rightarrow \mathbb{R}$ is comparison meaningful (or invariant if $E=[0,1]$ ).

Let $\mathcal{B}\left(E^{n}\right)$ be the class of all minimal invariant subsets of $E^{n}$, and define

$$
B_{x}(E):=\{\phi(x) \mid \phi \in \Phi(E)\}
$$

for all $x \in E^{n}$. Then, we have

$$
\mathcal{B}\left(E^{n}\right)=\left\{B_{x}(E) \mid x \in E^{n}\right\}
$$

which clearly shows that the elements of $\mathcal{B}\left(E^{n}\right)$ partition $E^{n}$ into equivalence classes, where $x, y \in E^{n}$ are equivalent if there exists $\phi \in \Phi(E)$ such that $y=\phi(x)$. A complete description of elements of $\mathcal{B}\left(E^{n}\right)$ is given in the following proposition.

Proposition $2.1([2,10])$. We have $B \in \mathcal{B}\left(E^{n}\right)$ if and only if there exists a permutation $\pi$ on $[n]$ and a sequence $\left\{\triangleleft_{i}\right\}_{i=0}^{n}$ of symbols $\triangleleft_{i} \in\{<,=\}$, containing at least one $<$ if $e_{0} \in E$ and $e_{1} \in E$, such that

$$
B=\left\{x \in E^{n} \mid e_{0} \triangleleft_{0} x_{\pi(1)} \triangleleft_{1} \cdots \triangleleft_{n-1} x_{\pi(n)} \triangleleft_{n} e_{1}\right\},
$$

where $\triangleleft_{0}$ is $<$ if $e_{0} \notin E$ and $\triangleleft_{n}$ is $<$ if $e_{1} \notin E$. 
Example 2.1. The unit square $[0,1]^{2}$ contains exactly eleven minimal invariant subsets, namely the open triangles $\left\{\left(x_{1}, x_{2}\right) \mid 0<x_{1}<x_{2}<1\right\}$ and $\left\{\left(x_{1}, x_{2}\right) \mid 0<x_{2}<x_{1}<1\right\}$, the open diagonal $\left\{\left(x_{1}, x_{2}\right) \mid 0<x_{1}=x_{2}<1\right\}$, the four square vertices, and the four open line segments joining neighboring vertices.

We also have the following important result.

Proposition $2.2([2,7,10])$. Consider a function $f: E^{n} \rightarrow E$.

i) If $f$ is idempotent (i.e., $f(x, \ldots, x)=x$ for all $x \in E$ ) and comparison meaningful, then it is invariant.

ii) If $f$ is invariant, then it is comparison meaningful.

iii) If $E$ is open, then $f$ is idempotent and comparison meaningful if and only if it is invariant.

iv) $f$ is invariant if and only if, for any $B \in \mathcal{B}\left(E^{n}\right)$, either $\left.f\right|_{B} \equiv c$ is a constant $c \in$ $\left\{e_{0}, e_{1}\right\} \cap E$ (if this constant exists) or there is $i \in[n]$ so that $\left.f\right|_{B}=\left.P_{i}\right|_{B}$ is the projection on the ith coordinate.

For nondecreasing invariant functions, a crucial role in their characterization is played by an equivalence relation $\sim$ acting on $\mathcal{B}\left(E^{n}\right)$, namely $B \sim C$ if and only if $P_{i}(B)=P_{i}(C)$ for all $i \in[n]$. Note that projections $P_{i}(B)$ of minimal invariant subsets are necessarily either $\left\{e_{0}\right\} \cap E$ or $\left\{e_{1}\right\} \cap E$ or $E^{\circ}$. Further, for any $B \in \mathcal{B}\left(E^{n}\right)$, the set

$$
B^{*}=\bigcup_{\substack{C \in \mathcal{B}\left(E^{n}\right) \\ C \sim B}} C=P_{1}(B) \times \cdots \times P_{n}(B)
$$

is an invariant subset of $E^{n}$, and

$$
\mathcal{B}^{*}\left(E^{n}\right)=\left\{B^{*} \mid B \in \mathcal{B}\left(E^{n}\right)\right\}
$$

is a partition of $E^{n}$ coarsening $\mathcal{B}\left(E^{n}\right)$. We also have $\operatorname{card}\left(\mathcal{B}^{*}\left(E^{n}\right)\right)=k^{n}$, where $k=$ $1+\operatorname{card}\left(E \cap\left\{e_{0}, e_{1}\right\}\right)$.

Notice that any subset $B^{*}$ can also be regarded as a minimal "strongly" invariant subset of $E^{n}$ in the sense that

$$
\left\{\left(\phi_{1}\left(x_{1}\right), \ldots, \phi_{n}\left(x_{n}\right)\right) \mid x \in B^{*}\right\} \subseteq B^{*} \quad\left(\phi_{1}, \ldots, \phi_{n} \in \Phi(E)\right) .
$$

Equivalently, the characteristic function $\mathbf{1}_{B^{*}}: E^{n} \rightarrow \mathbb{R}$ is strongly comparison meaningful.

From the natural order

$$
\left\{e_{0}\right\} \prec E^{\circ} \prec\left\{e_{1}\right\}
$$

we can straightforwardly derive a partial order $\preceq$ on $\mathcal{B}\left(E^{n}\right)$, namely $B \preceq C$ if and only if $P_{i}(B) \preceq P_{i}(C)$ for all $i \in[n]$. A partial order on $\mathcal{B}^{*}\left(E^{n}\right)$ can be defined similarly.

Denote by $\mathcal{M}_{n}$ the system of all nondecreasing functions $\mu:\{0,1\}^{n} \rightarrow\{0,1\}$, and let

$$
\mathcal{M}_{n}(E):=\mathcal{M}_{n} \backslash\left\{\mu_{j} \mid j \in\{0,1\}, e_{j} \notin E\right\},
$$

where $\mu_{j} \in \mathcal{M}_{n}$ is the constant set function $\mu_{j} \equiv j$. Clearly $\mathcal{M}_{n}(E)$ is partially ordered through the order defined as

$$
\mu \preceq \mu^{\prime} \quad \Leftrightarrow \quad \mu(x) \leq \mu^{\prime}(x) \forall x \in\{0,1\}^{n} .
$$


For $\mu \in \mathcal{M}_{n}(E)$, we define a function $L_{\mu}: E^{n} \rightarrow E$ by

$$
L_{\mu}\left(x_{1}, \ldots, x_{n}\right)=\bigvee_{\substack{t \in\{0,1\}^{n} \\ \mu(t)=1}} \bigwedge_{t_{i}=1} x_{i}
$$

with obvious conventions

$$
\bigvee_{\varnothing}=e_{0} \quad \text { and } \quad \bigwedge_{\varnothing}=e_{1}
$$

Observe that for any $\mu \in \mathcal{M}_{n}(E), L_{\mu}$ is a continuous invariant function which is also idempotent whenever $\mu(0, \ldots, 0)<\mu(1, \ldots, 1)$, that is, whenever $\mu(0, \ldots, 0)=0$ and $\mu(1, \ldots, 1)=1$.

Remark. Functions $\mu \in \mathcal{M}_{n}(E)$ with $\mu(0, \ldots, 0)<\mu(1, \ldots, 1)$ are called also $\{0,1\}$ valued fuzzy measures (when an element $t \in\{0,1\}^{n}$ is taken as the characteristic vector of a subset of $[n])$. For any such $\mu$, the corresponding function $L_{\mu}$ is exactly the Choquet integral with respect to $\mu[4,13]$, but also the Sugeno integral with respect to $\mu[15,13]$. These functions are called also lattice polynomials [3] or Boolean max-min functions [8].

We also have the following result.

Proposition $2.3([7,10])$. Consider a function $f: E^{n} \rightarrow E$. Then we have

i) $f$ is continuous and invariant if and only if $f=L_{\mu}$ for some $\mu \in \mathcal{M}_{n}(E)$.

ii) $f$ is nondecreasing and invariant if and only if there exists a nondecreasing mapping $\xi: \mathcal{B}^{*}\left(E^{n}\right) \rightarrow \mathcal{M}_{n}(E)$ so that

$$
f(x)=L_{\xi\left(B^{*}\right)}(x) \quad\left(x \in B^{*} \in \mathcal{B}^{*}\left(E^{n}\right)\right) .
$$

\section{Comparison meaningful functions}

Following Definition 2.1, the invariance of a function $f: E^{n} \rightarrow E$ can be reduced to the invariance of $\left.f\right|_{B}$ for all minimal invariant subsets $B \in \mathcal{B}\left(E^{n}\right)$. This observation is a key point in the description of invariant functions as given in Proposition 2.2, iv). However, in the case of comparison meaningful functions, the situation is more complicated. In fact, we have to examine property (1) for $x \in B, y \in C$, with $B, C \in \mathcal{B}\left(E^{n}\right)$, to be able to describe comparison meaningful functions. We start first with the case when $B=C$, i.e., when $y=\phi(x)$ for some $\phi \in \Phi(E)$.

Proposition 3.1. Let $f: E^{n} \rightarrow \mathbb{R}$ be a comparison meaningful function. Then, for any $B \in \mathcal{B}\left(E^{n}\right)$, there is an index $i_{B} \in[n]$ and a strictly monotone or constant function $g_{B}: P_{i_{B}}(B) \rightarrow \mathbb{R}$ such that

$$
f(x)=g_{B}\left(x_{i_{B}}\right) \quad\left(x=\left(x_{1}, \ldots, x_{n}\right) \in B\right) .
$$

Proof. Observe first that for any $B \in \mathcal{B}\left(E^{n}\right)$, the cardinality

$$
\operatorname{card}\left(\left\{x_{1}, \ldots, x_{n}\right\} \cap E^{\circ}\right)
$$

is constant for all $x=\left(x_{1}, \ldots, x_{n}\right) \in B$. 
Denote this cardinality as $m_{B}$. Evidently, $m_{B}=0$ if and only if $B$ is a singleton and then Proposition 3.1 obviously holds. For $m_{B}>0$, i.e., $m_{B} \in[n]$, the set $B$ equipped with standard Cartesian partial order $\leq$ is isomorphic to the set

$$
I_{m_{B}}:=\left\{\left(u_{1}, \ldots, u_{m_{B}}\right) \in\right] 0,1\left[{ }^{m_{B}} \mid u_{1}<\cdots<u_{m_{B}}\right\}
$$

equipped with $\leq$. Therefore, the comparison meaningfulness of $f$ on $B$ can be isomorphically translated to the study of comparison meaningful functions on $I_{m_{B}}$. Evidently, if $m_{B}=1$, Proposition 3.1 holds (on $I_{1}$ ) for comparison meaningful function $\left.f:\right] 0,1[\rightarrow \mathbb{R}$ which, if nonconstant, necessarily should be strictly monotone.

Now, suppose that $m_{B}=m \geq 2$ and $f: I_{m} \rightarrow \mathbb{R}$ is a comparison meaningful function. Fix an element $u=\left(u_{1}, \ldots, u_{m}\right) \in I_{m}$ and, for $i \in[m]$, define

$$
u^{i}(x):=\left(u_{1}, \ldots, u_{i-1}, x, u_{i+1}, \ldots, u_{m}\right) \quad(x \in] u_{i-1}, u_{i+1}[),
$$

with the convention that $u_{0}:=0$ and $u_{m+1}:=1$. Then obviously all $u^{i}(x)$ are elements of $I_{m}$. We also denote $u^{i}:=u^{i}\left(\frac{u_{i}+u_{i+1}}{2}\right)$.

We now have three exclusive cases to consider:

1. Suppose that all $f\left(u^{i}\right)$ equal $f(u)$. Then we can show that $f \equiv f(u)$ on whole domain $I_{m}$, proving our claim. We proceed in two steps:

(a) We have $f(u)=f\left(u^{i}(x)\right)$ for all $i \in[m]$ and all $\left.x \in\right] u_{i-1}, u_{i+1}[$. Indeed, let $i \in[m]$ and $\left.x^{\prime}, x^{\prime \prime} \in\right] u_{i-1}, u_{i+1}\left[\right.$ such that $x^{\prime}<x^{\prime \prime}$. There is $\phi \in \Phi(] 0,1[)$ such that $\phi\left(u_{i}\right)=x^{\prime}, \phi\left(u_{i}^{i}\right)=x^{\prime \prime}$, and $\phi\left(u_{j}\right)=u_{j}$ for all $j \neq i$. By comparison meaningfulness of $f$, we have $f\left(u^{i}\left(x^{\prime}\right)\right)=f\left(u^{i}\left(x^{\prime \prime}\right)\right)$.

(b) Let $v \in I_{m}$. There is $\phi \in \Phi(] 0,1[)$ such that $\phi(u)=v$ and $\phi\left(u^{i}\right)=v^{i}$ for all $i \in[m]$. By comparison meaningfulness of $f$, we have $f(v)=f\left(v^{i}\right)$ for all $i \in[m]$ and, as in step (a), $f(v)=f\left(v^{i}(x)\right)$ for all $i \in[m]$ and all $\left.x \in\right] v_{i-1}, v_{i+1}[$.

As $u$ and $v$ can always be joined by a polygonal line consisting entirely of line segments in $I_{m}$ that are parallel to the coordinate axes, we conclude that $f(v)=f(u)$.

2. Suppose that all but one $f\left(u^{i}\right)$ equal $f(u)$, say only for $k$ we have $f\left(u^{k}\right) \neq f(u)$. Then, using the same reasoning as in case 1 , it is easy to see that $f$ depends only on $k$ th coordinate and hence $f(v)=g\left(v_{k}\right)$ for any $v \in I_{m}$, where $g$ is strictly monotone (decreasing if $f\left(u^{k}\right)<f(u)$, increasing in the opposite case), again proving our claim. Indeed, as there is $\phi \in \Phi(] 0,1[)$ such that $\phi(u)=v$ and $\phi\left(u^{k}\right)=v^{k}$, by comparison meaningfulness of $f$, we have

$$
f(u)\left\{\begin{array}{c}
< \\
>
\end{array}\right\} f\left(u^{k}\right) \Rightarrow f(v)\left\{\begin{array}{c}
< \\
>
\end{array}\right\} f\left(v^{k}\right) .
$$

3. It remains to show that it is impossible that for two different indices $i, j \in[m]$ we have $f\left(u^{i}\right) \neq f(u)$ and $f\left(u^{j}\right) \neq f(u)$. Denote by $A$ the set of all elements from $I_{m}$ with fixed coordinates $x_{k}=u_{k}$ for all $k \in[m] \backslash\{i, j\}$. Consider also the following subsets

$$
\begin{aligned}
& A_{1}=\left\{x \in A \mid x_{i}<u_{i} \text { and } x_{j}>u_{j}\right\}, \\
& A_{2}=\left\{x \in A \mid x_{i}>u_{i} \text { and } x_{j}<u_{j}\right\}, \\
& A_{3}=\left\{x \in A \mid x_{i}<u_{i} \text { and } x_{j}<u_{j}\right\}, \\
& A_{4}=\left\{x \in A \mid x_{i}>u_{i} \text { and } x_{j}>u_{j}\right\} .
\end{aligned}
$$


There are four different situations to discuss depending on the type of inequalities occurring in above mentioned cases:

(a) Suppose $f\left(u^{i}\right)>f(u)$ and $f\left(u^{j}\right)>f(u)$. Then, by comparison meaningfulness, $f$ is strictly increasing along the line segment connecting $u$ and $u^{i}$ and along the line segment connecting $u$ and $u^{j}$.

i. If $f(v)<f(u)$ for some $v \in A_{1}$ then each $z \in A_{1}$ can be obtained as $\phi(v)$ for some $\phi \in \Phi(] 0,1[)$ such that $\phi(u)=u$ and hence, by comparison meaningfulness of $f$, we have $f(z)<f(u)$ for all $z \in A_{1}$. Now, for any sequence $\left(z_{n}\right)$ of $A_{1}$, with $\lim z_{n}=u^{j}$, we have $f\left(z_{n}\right)<f(u)<f\left(u^{j}\right)$ and thus $u^{j}$ is a discontinuity of $f$. It follows that, for any $\phi \in \Phi(] 0,1[)$, we have $\lim \phi\left(z_{n}\right)=\phi\left(u^{j}\right)$ and $f\left(\phi\left(z_{n}\right)\right)<f(\phi(u))<f\left(\phi\left(u^{j}\right)\right)$, i.e., $\phi\left(u^{j}\right)$ is also a discontinuity of $f$. This means that any point in $I_{m}$ is a discontinuity of $f$ (any point in $I_{m}$ is such a $\phi\left(u^{j}\right)$ for a suitable $\phi$ ), which is impossible.

ii. If $f(v)=f(u)$ for some $v \in A_{1}$ then we have $f(z)=f(u)$ for all $z \in A_{1}$. It follows that $u^{j}$ is a discontinuity of $f$ and we conclude as in the previous subcase.

iii. If $f(v)>f(u)$ for some $v \in A_{1}$ then we have $f(z)>f(u)$ for all $z \in A_{1}$ and, by comparison meaningfulness, the opposite inequality holds for all elements from $A_{2}$. Now, we can easily show that $u^{i}$ is a discontinuity and then we arrive at a contradiction as previously.

(b) If $f\left(u^{i}\right)<f(u)$ and $f\left(u^{j}\right)<f(u)$ we use exactly the same reasoning as in the previous case.

(c) Suppose $f\left(u^{i}\right)<f(u)$ and $f\left(u^{j}\right)>f(u)$. Then either $f(z)=f(u)$ for all $z \in A_{4}$ (then both $u^{i}$ and $u^{j}$ are discontinuities), or $f(z)>f(u)$ for all $z \in A_{4}$ (then $u^{i}$ is discontinuity), or $f(z)<f(u)$ for all $z \in A_{4}$ (then $u^{j}$ is discontinuity). In either case we arrive at a contradiction.

(d) If $f\left(u^{i}\right)>f(u)$ and $f\left(u^{j}\right)<f(u)$ we use exactly the same reasoning as in the previous case.

As an easy corollary of Proposition 3.1 we obtain the characterization of invariant functions stated in Proposition 2.2, iv); see also [2]. Indeed, for a fixed $B \in \mathcal{B}\left(E^{n}\right)$, we should have $f(x)=g\left(x_{i}\right)$ and hence, for all $\phi \in \Phi(E)$ with fixed point $x_{i}$, we have

$$
\phi\left(g\left(x_{i}\right)\right)=\phi(f(x))=f(\phi(x))=g\left(x_{i}\right),
$$

which implies that $g\left(x_{i}\right)$ is a fixed point of all such $\phi$ 's, that is,

$$
g\left(x_{i}\right)=x_{i} \text { or } e_{0} \text { or } e_{1}
$$

As we have already observed, the structure of invariant functions on a given minimal invariant subset is completely independent of their structure on any other minimal invariant subset. This fact is due to the invariance property: $\phi(x) \in B$ for all $x \in B, \phi \in \Phi(E)$ and $B \in \mathcal{B}\left(E^{n}\right)$. However, in the case of comparison meaningful functions we are faced a quite different situation, in which we should take into account all minimal invariant subsets. 
Observe first that for a given comparison meaningful function $f: E^{n} \rightarrow \mathbb{R}$ and a given $B \in \mathcal{B}\left(E^{n}\right)$, the corresponding index $i_{B}$ need not be determined univocally. This happens for instance when $g_{B}$ is constant or when $B$ is defined with equalities on coordinates (see Proposition 2.1). On the other hand, given $i_{B}$, the function $g_{B}$ is necessarily unique.

Now, we are ready to give a complete description of all comparison meaningful functions.

Theorem 3.1. The function $f: E^{n} \rightarrow \mathbb{R}$ is comparison meaningful if and only if, for any $B \in \mathcal{B}\left(E^{n}\right)$, there exist an index $i_{B} \in[n]$ and a strictly monotone or constant mapping $g_{B}: P_{i_{B}}(B) \rightarrow \mathbb{R}$ such that

$$
f(x)=g_{B}\left(x_{i_{B}}\right) \quad(x \in B),
$$

where, for any $B, C \in \mathcal{B}\left(E^{n}\right)$, either $g_{B}=g_{C}$, or $\operatorname{Ran}\left(g_{B}\right)=\operatorname{Ran}\left(g_{C}\right)$ is singleton, or $\operatorname{Ran}\left(g_{B}\right)<\operatorname{Ran}\left(g_{C}\right)$, or $\operatorname{Ran}\left(g_{B}\right)>\operatorname{Ran}\left(g_{C}\right)$. (Note that $\operatorname{Ran}\left(g_{B}\right)<\operatorname{Ran}\left(g_{C}\right)$ means that for all $r \in \operatorname{Ran}\left(g_{B}\right)$ and all $s \in \operatorname{Ran}\left(g_{C}\right)$, we have $r<$ s.)

Proof. (Sufficiency) Let $B, C \in \mathcal{B}\left(E^{n}\right), x \in B, y \in C$, and $\phi \in \Phi(E)$. We have three cases to consider:

1. If $B=C$ then whether $g_{B}$ be strictly monotone or constant, we have

$$
\begin{aligned}
f(x) \leq f(y) & \Leftrightarrow g_{B}\left(x_{i_{B}}\right) \leq g_{B}\left(y_{i_{B}}\right) \\
& \Leftrightarrow g_{B}\left(\phi\left(x_{i_{B}}\right)\right) \leq g_{B}\left(\phi\left(y_{i_{B}}\right)\right) \\
& \Leftrightarrow f(\phi(x)) \leq f(\phi(y)) .
\end{aligned}
$$

2. If $B \neq C$ and either $g_{B}=g_{C}$ or $\operatorname{Ran}\left(g_{B}\right)=\operatorname{Ran}\left(g_{C}\right)$ is singleton, then we conclude immediately as in the previous case.

3. The case $B \neq C$ and $\operatorname{Ran}\left(g_{B}\right)<\operatorname{Ran}\left(g_{C}\right)$ is immediate.

(Necessity) Due to Proposition 3.1 we have ensured the existence of indexes $i_{B}$ (not necessarily unique) and the existence of comparison meaningful functions $g_{B}: P_{i_{B}}(B) \rightarrow \mathbb{R}$ representing $f$ in the form $(2)$.

Now, suppose that $B, C \in \mathcal{B}\left(E^{n}\right), B \neq C$. We have three cases to consider:

1. If $\operatorname{Ran}\left(g_{B}\right)$ and $\operatorname{Ran}\left(g_{C}\right)$ are singletons, then the result holds trivially.

2. Assume $\operatorname{Ran}\left(g_{B}\right)$ is not singleton and $\operatorname{Ran}\left(g_{C}\right)$ is singleton and choose $u \in E^{\circ}$.

(a) If $g_{B}(u)=g_{C}$ then comparison meaningfulness ensures $\operatorname{Ran}\left(g_{B}\right)=\operatorname{Ran}\left(g_{C}\right)$ is singleton, a contradiction.

(b) If, say, $g_{B}(u)<g_{C}$ then comparison meaningfulness ensures $\operatorname{Ran}\left(g_{B}\right)<\operatorname{Ran}\left(g_{C}\right)$.

3. Assume $\operatorname{Ran}\left(g_{B}\right)$ and $\operatorname{Ran}\left(g_{C}\right)$ are not singletons and choose $u \in E^{\circ}$.

(a) If $g_{B}(u)=g_{C}(u)$ then comparison meaningfulness ensures $g_{B}=g_{C}$.

(b) If, say, $g_{B}(u)<g_{C}(u)$ then this inequality also holds for all $v \in E^{\circ}$ and if, for some $v, w \in E^{\circ}$ we have $g_{C}(v) \leq g_{B}(w)$, then, depending on whether $w>v$ or oppositely, due to comparison meaningfulness, this inequality holds for all $z>v$ (or oppositely), i.e., we have $g_{B}(v)<g_{C}(v) \leq g_{B}\left(v^{+}\right)$(or $g_{B}\left(v^{-}\right)$if it was $w<v)$, hence in any case, $g_{B}$ is not continuous at $v$, and due to comparison meaningfulness, the same is true for all points in $E^{\circ}$. However, because of the monotonicity of $g_{B}$, this is impossible, proving $\operatorname{Ran}\left(g_{B}\right)<\operatorname{Ran}\left(g_{C}\right)$. 
Example 3.1. Put $E=[0,1]$ and $n=2$. Then there are eleven minimal invariant subsets in $\mathcal{B}\left([0,1]^{2}\right)$, namely $B_{1}=\{(0,0)\}, B_{2}=\{(1,0)\}, B_{3}=\{(1,1)\}, B_{4}=\{(0,1)\}, B_{5}=$ ] $0,1\left[\times\{0\}, B_{6}=\{1\} \times\right] 0,1\left[, B_{7}=\right] 0,1\left[\times\{1\}, B_{8}=\{0\} \times\right] 0,1\left[, B_{9}=\left\{\left(x_{1}, x_{2}\right) \mid 0<x_{1}=\right.\right.$ $\left.x_{2}<1\right\}, B_{10}=\left\{\left(x_{1}, x_{2}\right) \mid 0<x_{1}<x_{2}<1\right\}, B_{11}=\left\{\left(x_{1}, x_{2}\right) \mid 0<x_{2}<x_{1}<1\right\}$. Let $i_{B_{j}}=1$ and $g_{B_{j}}(x)=1-x$ for $j \in\{1,2,3,5,6,9,11\}$, and $i_{B_{j}}=2$ and $g_{B_{j}}(x)=2 x-3$ for $j \in\{4,7,8,10\}$, where always $x \in P_{i_{B_{j}}}\left(B_{j}\right)$. Then the relevant comparison meaningful function $f:[0,1]^{2} \rightarrow[0,1]$ is given by

$$
f\left(x_{1}, x_{2}\right)= \begin{cases}1-x_{1}, & \text { if } x_{1} \geq x_{2} \\ 2 x_{2}-3, & \text { if } x_{1}<x_{2}\end{cases}
$$

Theorem 3.1 enables us to characterize strong comparison meaningful functions, too. Observe that while in the case of comparison meaningful functions, for any point $x \in E^{n}$ the set of all $\phi(x)=\left(\phi\left(x_{1}\right), \ldots, \phi\left(x_{n}\right)\right)$, with $\phi \in \Phi(E)$, gives some minimal invariant set $B$, in the case of strong comparison meaningful functions we are faced to the set of all points $\left(\phi_{1}\left(x_{1}\right), \ldots, \phi_{n}\left(x_{n}\right)\right)$, with $\phi_{1}, \ldots, \phi_{n} \in \Phi(E)$, which is exactly the invariant set $B^{*}$ linked to the previous $B$, which together with Theorem 3.1 results in the next corollary.

Corollary 3.1. The function $f: E^{n} \rightarrow \mathbb{R}$ is strongly comparison meaningful if and only if, for any $B^{*} \in \mathcal{B}^{*}\left(E^{n}\right)$, there exist an index $i_{B^{*}} \in[n]$ and a strictly monotone or constant mapping $g_{B^{*}}: P_{i_{B^{*}}}\left(B^{*}\right) \rightarrow \mathbb{R}$ such that

$$
f(x)=g_{B^{*}}\left(x_{i_{B^{*}}}\right) \quad\left(x \in B^{*}\right),
$$

where, for any $B^{*}, C^{*} \in \mathcal{B}^{*}\left(E^{n}\right)$, either $g_{B^{*}}=g_{C^{*}}$, or $\operatorname{Ran}\left(g_{B^{*}}\right)=\operatorname{Ran}\left(g_{C^{*}}\right)$ is singleton, or $\operatorname{Ran}\left(g_{B^{*}}\right)<\operatorname{Ran}\left(g_{C^{*}}\right)$, or $\operatorname{Ran}\left(g_{B^{*}}\right)>\operatorname{Ran}\left(g_{C^{*}}\right)$.

\section{Monotone comparison meaningful functions}

In this section we will examine monotone comparison meaningful functions. Note that the monotonicity of a fusion function is a rather natural property.

Now, for any strictly monotone or constant real function $h: \mathbb{R} \rightarrow \mathbb{R}$, and any comparison meaningful function $f: E^{n} \rightarrow \mathbb{R}$, also the composite $h \circ f: E^{n} \rightarrow \mathbb{R}$ is comparison meaningful. Consequently, to get a complete description of monotone comparison meaningful functions it is enough to examine nondecreasing comparison meaningful functions only.

Theorem 4.1. Let $f: E^{n} \rightarrow \mathbb{R}$ be a nondecreasing function. Then $f$ is comparison meaningful if and only if it has the representation

$$
\left\{\left(i_{B}, g_{B}\right) \mid B \in \mathcal{B}\left(E^{n}\right)\right\}
$$

as stated in Theorem 3.1, such that any $g_{B}$ is either constant or strictly increasing, $\operatorname{Ran}\left(g_{B}\right)=$ $\operatorname{Ran}\left(g_{C}\right)$ if $B \sim C$, and $\operatorname{Ran}\left(g_{B}\right) \ngtr \operatorname{Ran}\left(g_{C}\right)$ if $B \nsim C$ and $B \preceq C$.

Proof. (Sufficiency) Easy, hence we omit it.

(Necessity) First of all, it is clear that no function $g_{B}$ can be strictly decreasing. 
Now, if $B \sim C$ and $B \neq C$, then there are $u, w \in B$ and $v \in C$ such that $u \leq v \leq w$, and hence $f(u) \leq f(v) \leq f(w)$. If follows immediately that it is not possible to have $\operatorname{Ran}\left(g_{B}\right)<\operatorname{Ran}\left(g_{C}\right)$ or $\operatorname{Ran}\left(g_{B}\right)>\operatorname{Ran}\left(g_{C}\right)$. Then, by Theorem 3.1, we necessarily have $g_{B}=g_{C}$, or $\operatorname{Ran}\left(g_{B}\right)=\operatorname{Ran}\left(g_{C}\right)$ is singleton.

If $B \nsim C$ and $B \preceq C$ then for all $u \in B$ and all $v \in C$ either $u \leq v$ or $u$ and $v$ are incomparable. Therefore we have either $g_{B}=g_{C}$, or $\operatorname{Ran}\left(g_{B}\right)=\operatorname{Ran}\left(g_{C}\right)$ is singleton, or $\operatorname{Ran}\left(g_{B}\right)<\operatorname{Ran}\left(g_{C}\right)$.

Now, several results mentioned in Section 2 are immediate corollaries of Theorems 3.1 and 4.1. Interesting seems to be also the next result, in which $\mathcal{G}(E)$ means the system of all strictly increasing or constant real functions $g$ defined either on $E^{\circ}$ or on singleton $\left\{e_{0}\right\} \cap E$ or on $\left\{e_{1}\right\} \cap E$ (if these singletons exist) and for $g_{1}, g_{2} \in \mathcal{G}(E)$ we put $g_{1} \preceq g_{2}$ if either $g_{1}=g_{2}$, or $\operatorname{Ran}\left(g_{1}\right)=\operatorname{Ran}\left(g_{2}\right)$ is a singleton, or $\operatorname{Ran}\left(g_{1}\right)<\operatorname{Ran}\left(g_{2}\right)$.

Corollary 4.1. A nondecreasing function $f: E^{n} \rightarrow \mathbb{R}$ is comparison meaningful if and only if there are nondecreasing mappings $\xi: \mathcal{B}^{*}\left(E^{n}\right) \rightarrow \mathcal{M}_{n}(E)$ and $\gamma: \mathcal{B}^{*}\left(E^{n}\right) \rightarrow \mathcal{G}(E)$ so that

$$
f(x)=\gamma\left(B^{*}\right)\left(L_{\xi\left(B^{*}\right)}(x)\right) \quad\left(x \in B^{*} \in \mathcal{B}^{*}\left(E^{n}\right)\right) .
$$

Proof. (Sufficiency) Easy.

(Necessity) Fix $B \in \mathcal{B}\left(E^{n}\right)$. If $g_{B}$ is constant then $g_{C}=g_{B}$ is constant for all $C \sim B$ and hence (3) holds with a constant function $\gamma\left(B^{*}\right)$ and $L_{\xi\left(B^{*}\right)}$ arbitrary.

On the other hand, if $g_{B}$ is strictly increasing then $g_{C}=g_{B}$ for all $C \sim B$. It follows that the function $g_{B}^{-1} \circ f: B^{*} \rightarrow E^{\circ}$ is well defined, nondecreasing, and invariant and hence, according to Proposition 2.3(ii), there exists $\mu \in \mathcal{M}_{n}(E)$ such that $g_{B}^{-1} \circ f=L_{\mu}$. Therefore, (3) holds with $\gamma\left(B^{*}\right)=g_{B}$ and $\xi\left(B^{*}\right)=\mu$.

Observe also that whenever $B^{*}$ is not singleton then the relevant function $\gamma\left(B^{*}\right)$ from the representation (3) can be obtained (for all $z \in E^{\circ}$ ) by

$$
\gamma\left(B^{*}\right)(z)=f\left(z_{1}, \ldots, z_{n}\right)
$$

where

$$
z_{i}= \begin{cases}e_{0}, & \text { if } P_{i}\left(B^{*}\right)=\left\{e_{0}\right\} \\ e_{1}, & \text { if } P_{i}\left(B^{*}\right)=\left\{e_{1}\right\} \\ z, & \text { otherwise }\end{cases}
$$

For example, if $E$ is open, then $\mathcal{B}^{*}\left(E^{n}\right)=\left\{E^{n}\right\}$ and then necessarily each monotone comparison meaningful $f: E^{n} \rightarrow \mathbb{R}$ is given by $f=g \circ L_{\mu}$, where $\mu \in \mathcal{M}_{n}(E)$ and $g(z)=f(z, \ldots, z)$ is strictly monotone or constant (see also [7]).

Based on Corollaries 3.1 and 4.1, we can characterize nondecreasing strong comparison meaningful functions as follows.

Corollary 4.2. A nondecreasing function $f: E^{n} \rightarrow \mathbb{R}$ is strongly comparison meaningful if and only if there is a mapping $\delta: \mathcal{B}^{*}\left(E^{n}\right) \rightarrow[n]$ and a nondecreasing mapping $\gamma: \mathcal{B}^{*}\left(E^{n}\right) \rightarrow$ $\mathcal{G}(E)$ such that

$$
f(x)=\gamma\left(B^{*}\right)\left(x_{\delta\left(B^{*}\right)}\right) \quad\left(x \in B^{*} \in \mathcal{B}^{*}\left(E^{n}\right)\right),
$$

where, if $\gamma\left(B^{*}\right)=\gamma\left(C^{*}\right)$, then also $\delta\left(B^{*}\right)=\delta\left(C^{*}\right)$ (unless $\gamma\left(B^{*}\right)=\gamma\left(C^{*}\right)$ is constant).

Continuity of a comparison meaningful function is even more restrictive and it forces the monotonicity. From Theorem 3.1 we have the next result (see also [7]). 
Corollary 4.3. A continuous function $f: E^{n} \rightarrow \mathbb{R}$ is comparison meaningful if and only if there is a continuous, strictly monotone or constant mapping $g: E \rightarrow \mathbb{R}$ and a function $\mu \in \mathcal{M}_{n}(E)$ such that

$$
f=g \circ L_{\mu} .
$$

Note that in trivial cases when $f$ is constant, $f$ admits also representations different from (4), however, always in the form $f=g \circ f^{*}$, where $g$ is a constant function on $E$ and $f^{*}: E^{n} \rightarrow E$ is an arbitrary function. In all other cases the representation (4) is unique.

Corollary 4.4. A continuous function $f: E^{n} \rightarrow \mathbb{R}$ is strongly comparison meaningful if and only if there is a continuous, strictly monotone or constant mapping $g: E \rightarrow \mathbb{R}$ and an index $i \in[n]$ so that

$$
f=g \circ P_{i}
$$

\section{Conclusion}

We have described the structure of a general comparison meaningful function. As corollaries, some results concerning special cases (monotone and/or continuous operators) were characterized. Moreover, our characterization can be understood also as a hint how to construct comparison meaningful operators.

\section{Acknowledgments}

This work was supported by two European actions (CEEPUS network SK-42 and COST action 274 TARSKI) as well as by grant VEGA 1/0273/03 and project APVT-20-046402.

\section{References}

[1] J. Aczél and F.S. Roberts, On the possible merging functions, Math. Social Sci. 17 (3) (1989) 205-243.

[2] L. Bartłomiejczyk and J. Drewniak, A characterization of sets and operations invariant under bijections, Aequationes Math. 68 (1) (2004) 1-9.

[3] G. Birkhoff, Lattice Theory, (Third Edition, AMS, Providence, 1967).

[4] G. Choquet, Theory of capacities, Ann. Inst. Fourier 5 (1953) 131-295.

[5] S.-R. Kim, On the possible scientific laws, Math. Social Sci. 20 (1) (1990) 19-36.

[6] R.D. Luce, D.H. Krantz, P. Suppes and A. Tversky, Foundations of measurement, volume III (Academic Press, New York, 1990).

[7] J.-L. Marichal, On order invariant synthesizing functions, J. Math. Psych. 46 (6) (2002) 661-676.

[8] J.-L. Marichal and P. Mathonet, On comparison meaningfulness of aggregation functions, J. of Math. Psych. 45 (2) (2001) 213-223. 
[9] J.-L. Marichal and M. Roubens, Characterization of some stable aggregation functions, Proc. 1st Int. Conf. on Industrial Engineering and Production Management (IEPM'93), Mons, Belgium, June 2-4, 1993, 187-196.

[10] R. Mesiar and T. Rückschlossová, Characterization of invariant aggregation operators, Fuzzy Sets and Systems 142 (1) (2004) 63-73.

[11] A. Orlov, The connection between mean quantities and admissible transformations, Math. Notes 30 (1981) 774-778.

[12] S. Ovchinnikov, Invariant functions on simple orders, Order 14 (4) (1998) 365-371.

[13] S. Ovchinnikov and A. Dukhovny, Integral representation of invariant functionals, $J$. Math. Anal. Appl. 244 (1) (2000) 228-232.

[14] F.S. Roberts, Measurement theory with applications to decision-making, utility and the social sciences (Addison-Wesley Pub., Reading, MA, 1979).

[15] M. Sugeno, Theory of fuzzy integrals and its applications, Ph.D. Thesis, Tokyo Institute of Technology, Tokyo, 1974.

[16] E. Yanovskaya, Group choice rules in problems with interpersonal preference comparisons, Automat. Remote Control 50 (6) (1989) 822-830.

\section{Addresses of the authors}

Jean-Luc MARICHAL, Faculty of Law, Economics, and Finance, University of Luxembourg, 162A, avenue de la Faïencerie, L-1511 Luxembourg, Grand Duchy of Luxembourg. Email: jeanluc.marichal[at]uni.lu

Radko MESIAR, Department of Mathematics, Slovak Technical University, Radlinského 11, 81368 Bratislava, Slovakia. Email: mesiar[at]vox.svf.stuba.sk

Tatiana RÜCKSCHLOSSOVÁ, Department of Mathematics, Slovak Technical University, Radlinského 11, 81368 Bratislava, Slovakia. Email: tatiana[at]vox.svf.stuba.sk 